\title{
THE HADRONISATION OF A QUARK-GLUON PLASMA
}

\author{
J. Cleymans ${ }^{1,2}$, K. Redlich ${ }^{2,3}$, H. Satz ${ }^{2,4}$ and E. Suhonen ${ }^{1,2,5}$
}

\begin{abstract}
We consider two scenarios for the expansion of a quark-gluon plasma. If the evolution is slow enough, the system can remain in equilibrium throughout its entire history up to the freeze-out of a hadron gas; for a very rapid expansion, it may break up into hadrons before or at the confinement transition, without ever going through an equilibrium hadron phase.

We compare hadron production rates in the two approaches and show that for a hadronisation temperature $T \simeq 200 \mathrm{MeV}$ and baryonic chemical potential $\mu_{B} \lesssim 500$ $\mathrm{MeV}$, their predictions essentially coincide. Present data on strange particle production lead to values in this range and hence cannot provide a distinction between the two scenarios.

Pion, nucleon and non-strange meson production seem to require a considerably lower freeze-out temperature and baryonic chemical potential. In the hadron gas picture, this is in accord with the difference in mean free path of the different hadrons in the medium; it suggests a sequential freeze-out, in which strange hadrons stop interacting earlier than non-strange hadrons.

In the quark-gluon plasma break-up, the hadronic final state fails to provide the high entropy per baryon observed in non-strange hadron production. The break-up moreover leads to a decrease of the entropy per baryon; hence it must be conceptually modified before it can be considered as a viable hadronisation mechanism.
\end{abstract}

1 Department of Physics, University of Cape Town, Rondebosch 7700, South Africa

2 Fakultät für Physik, Universität Bielefeld, D-4800 Bielefeld 1, Germany

3 Institute for Theoretical Physics, University of Wroclaw, PL-50205 Wroclaw, Poland

4 Theory Division, CERN, CH-1211 Geneva 23, Switzerland

5 Department of Theoretical Physics, University of Oulu, SF-90550 Oulu, Finland

CERN-TH 6759/92

BI-TP $92 / 55$

December 1992 


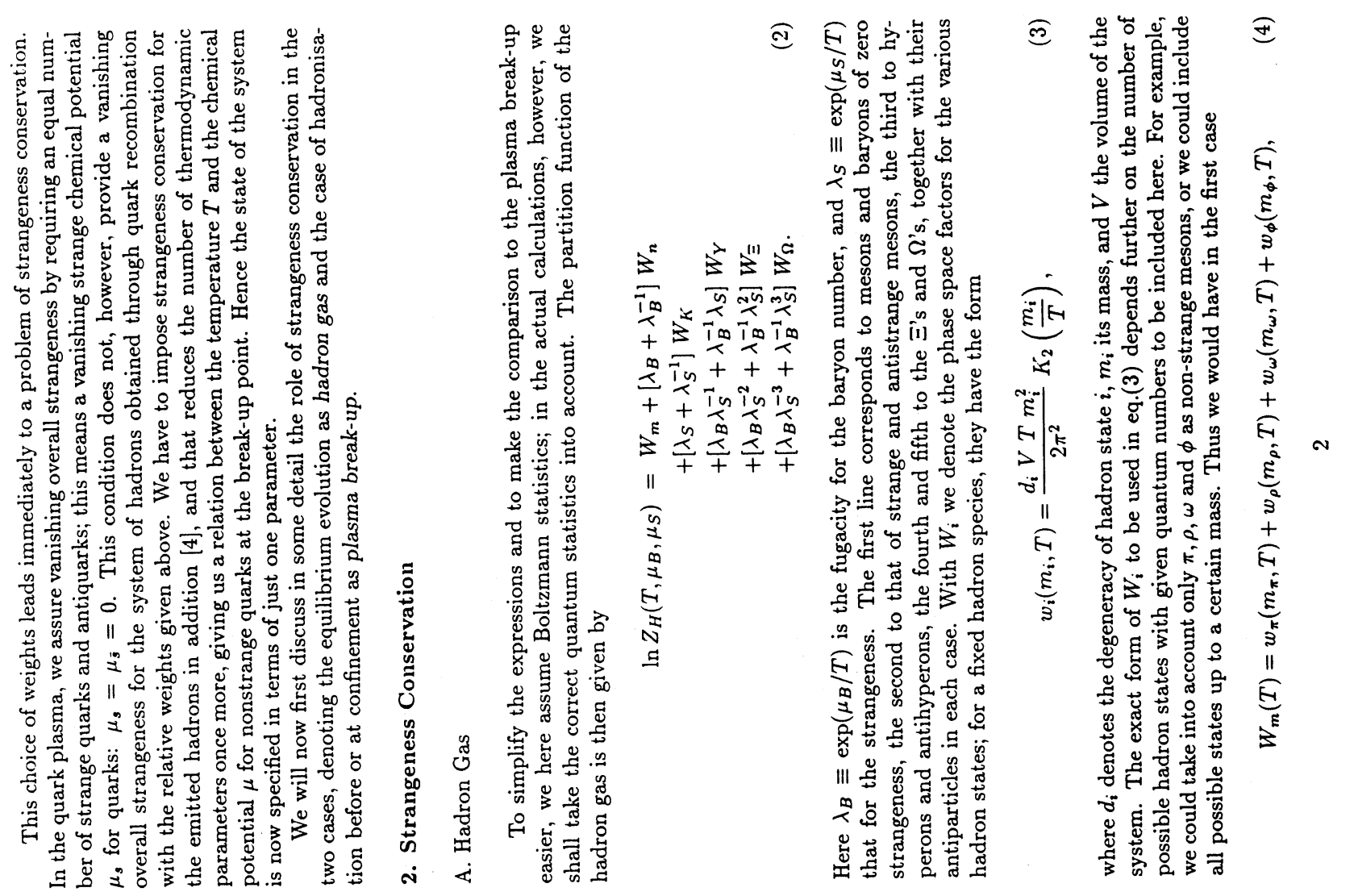

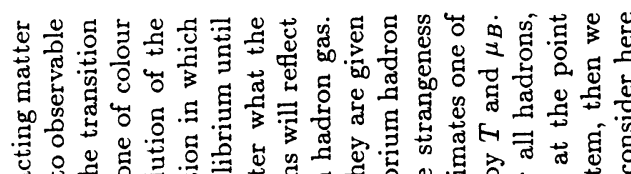

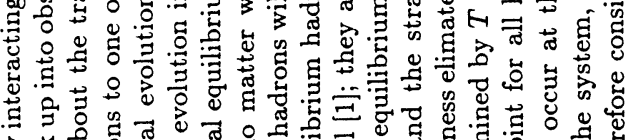

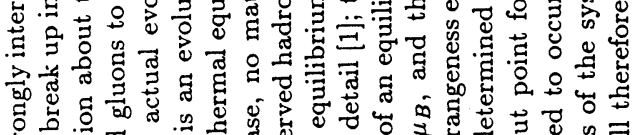

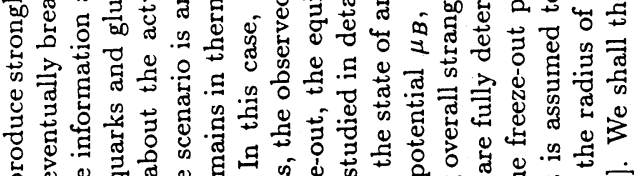

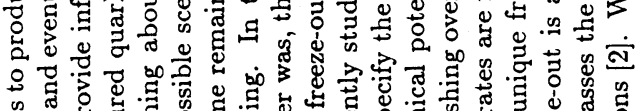

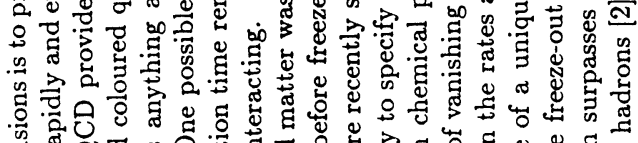

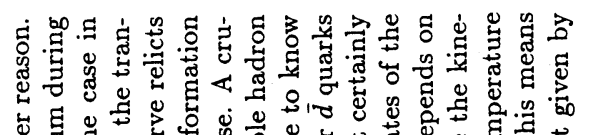

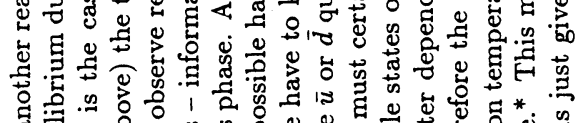
min Un Why In⿴囗十) MIn⿴囗十) Un

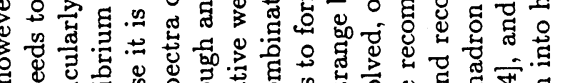
OIn

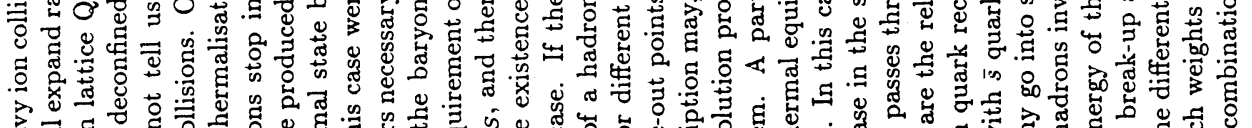
M. MM

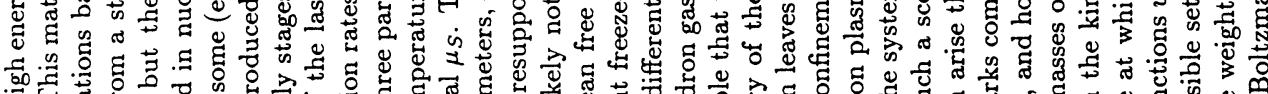

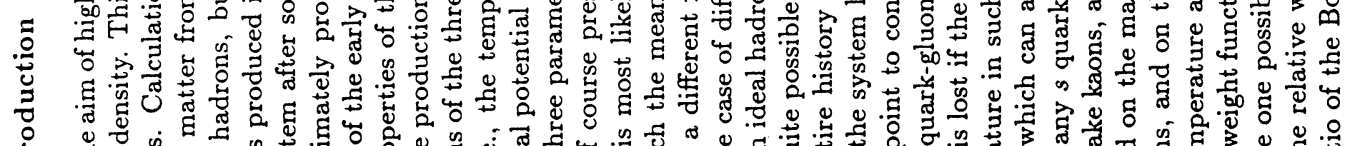

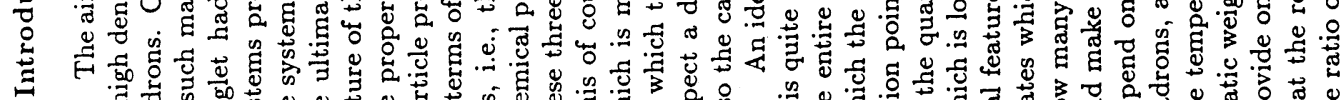

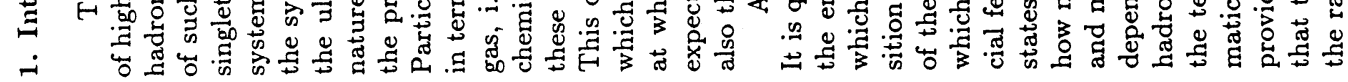

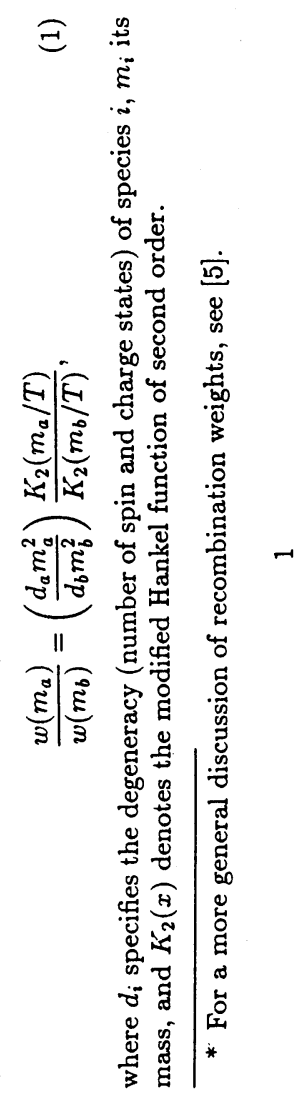



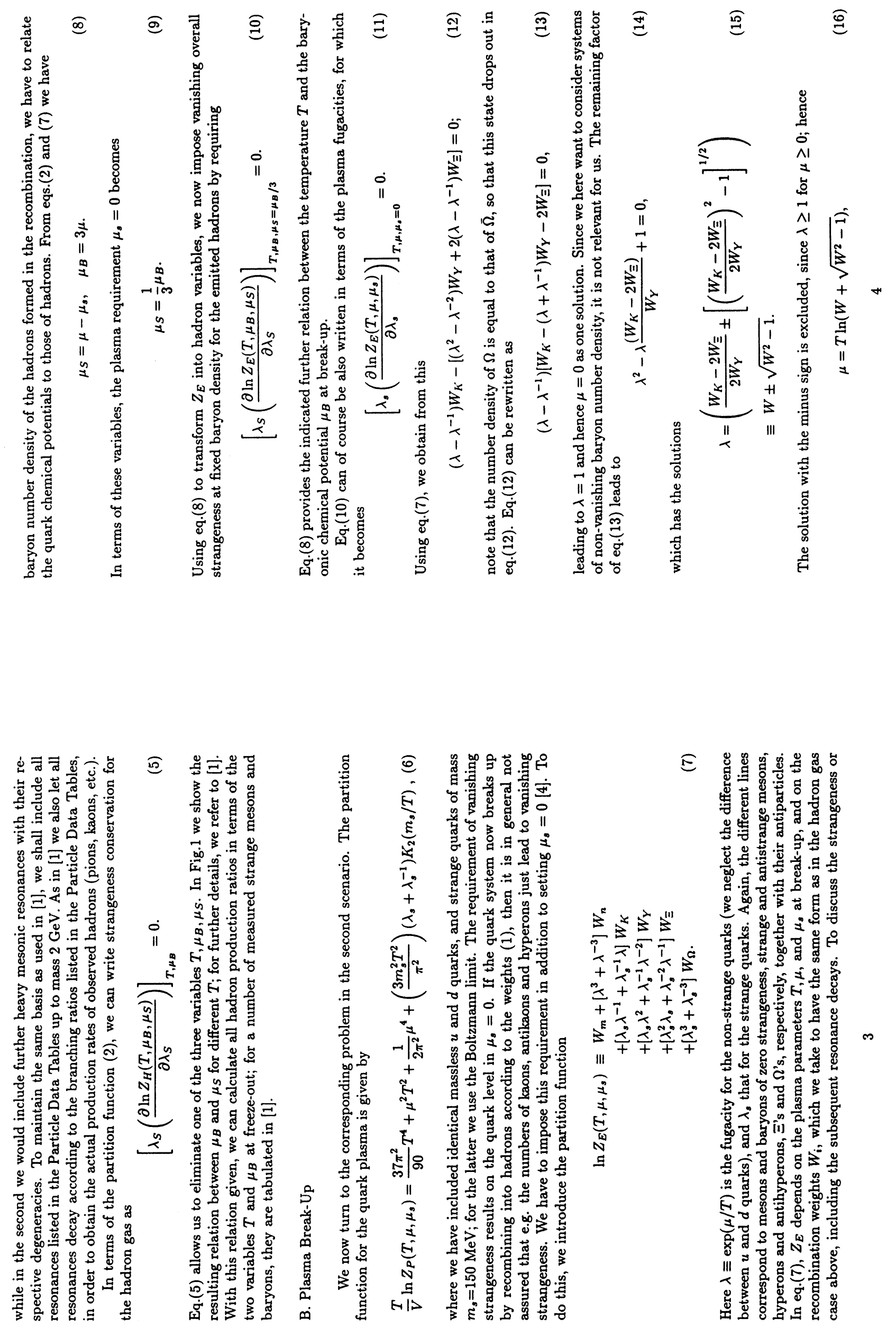


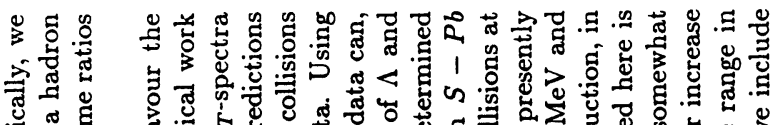

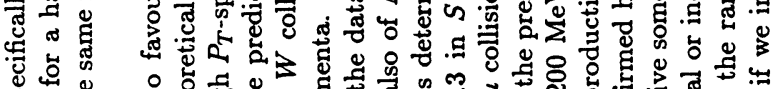

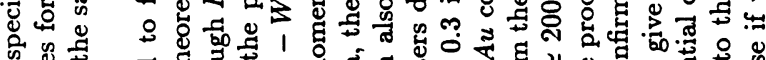

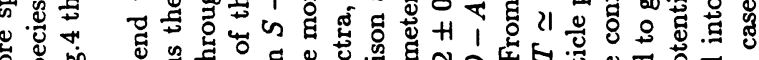
它

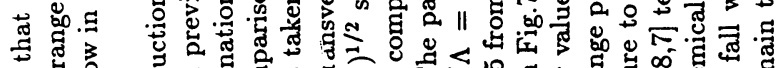

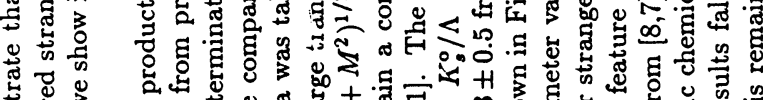

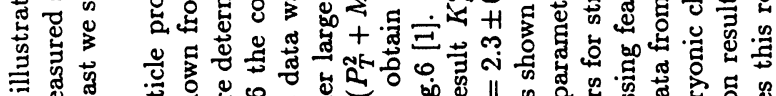
은 은

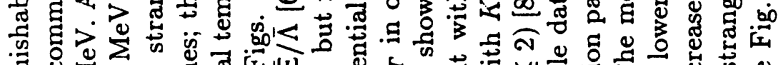
等。

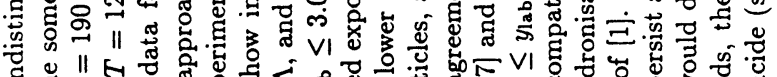
年

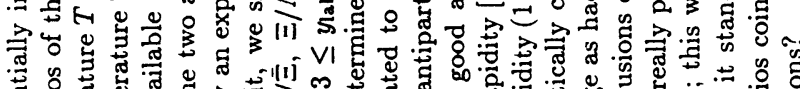

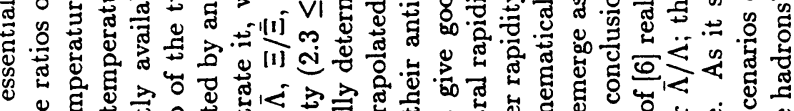

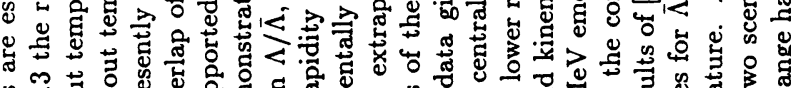

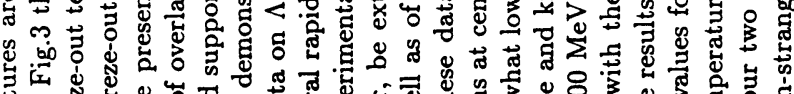

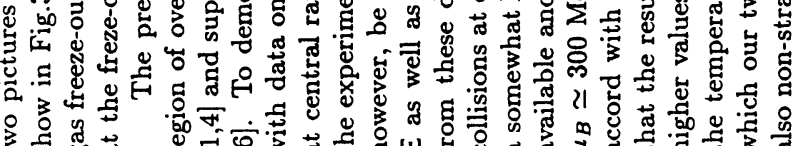

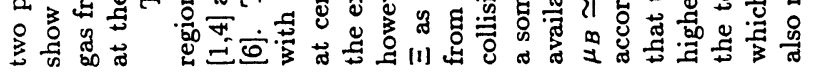

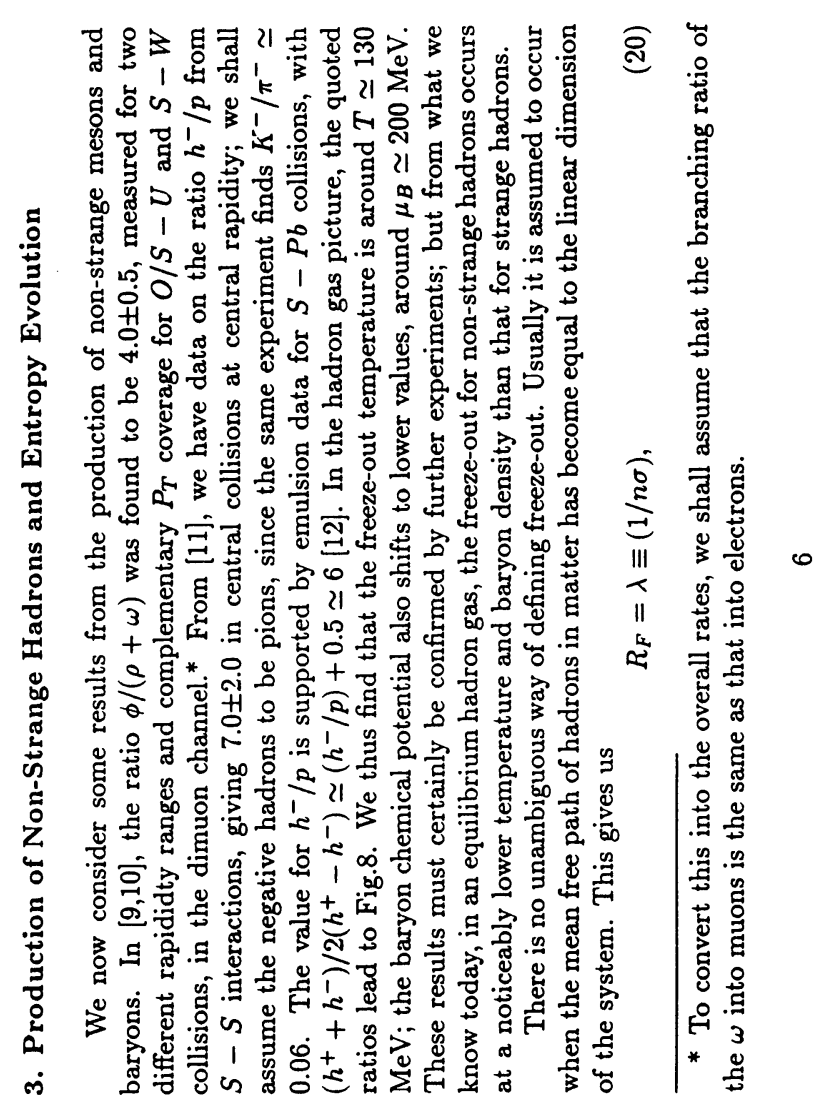

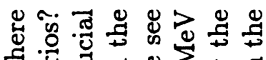

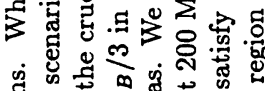

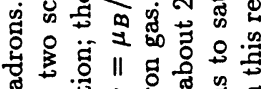

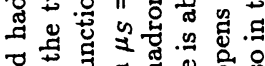

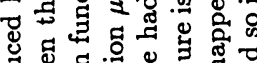

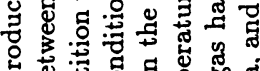

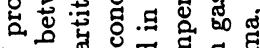

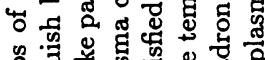

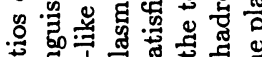
通.

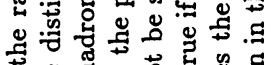

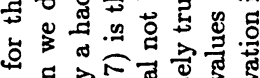

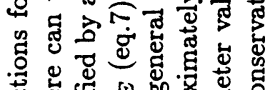

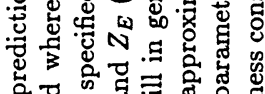

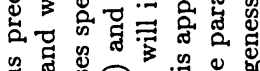

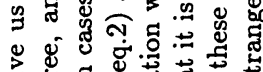

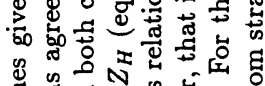

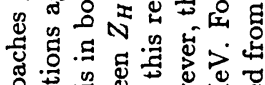

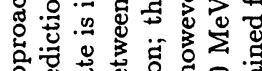

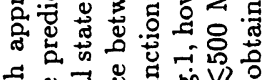

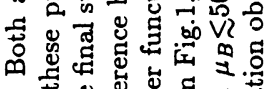

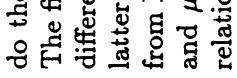



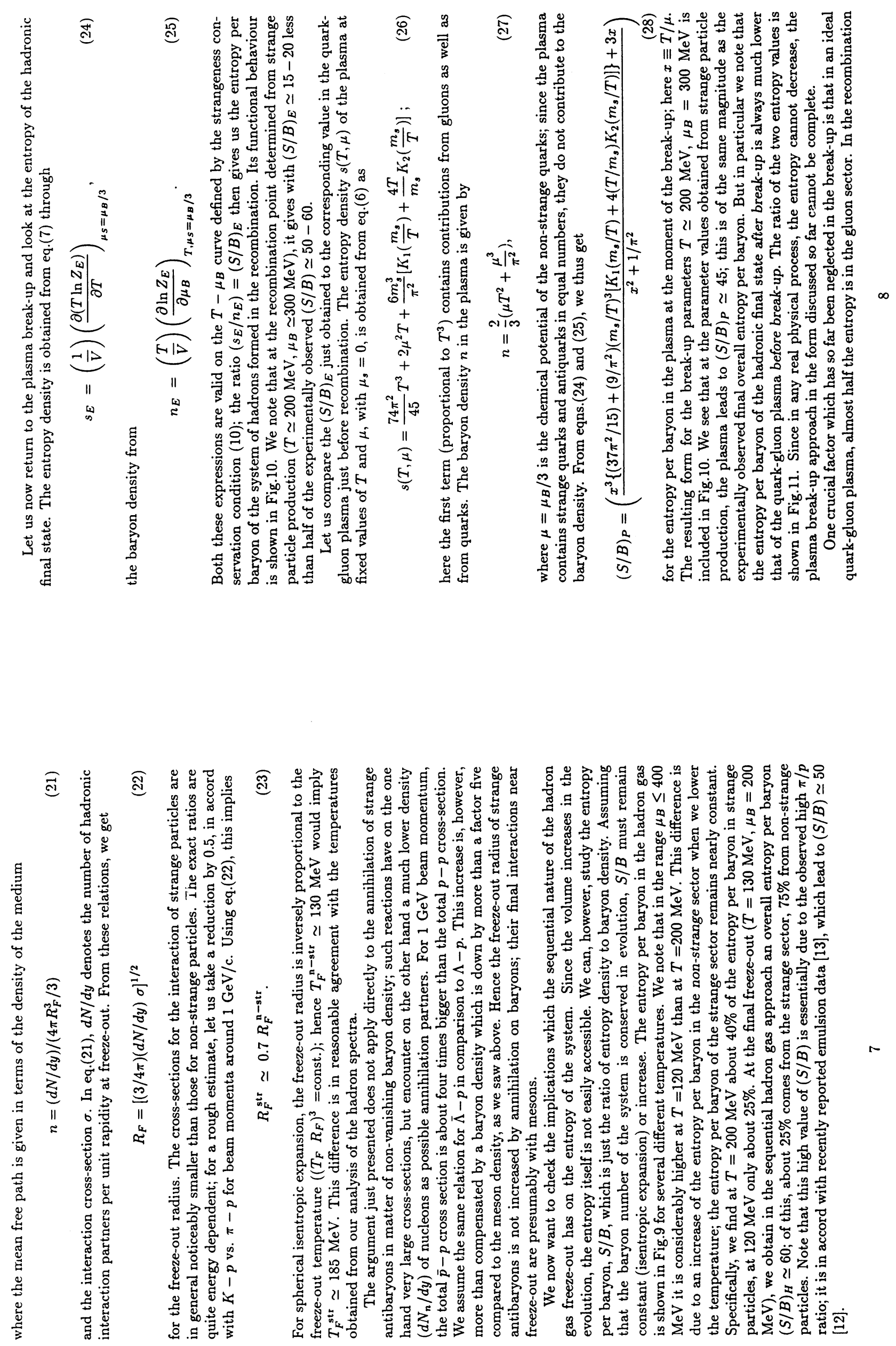

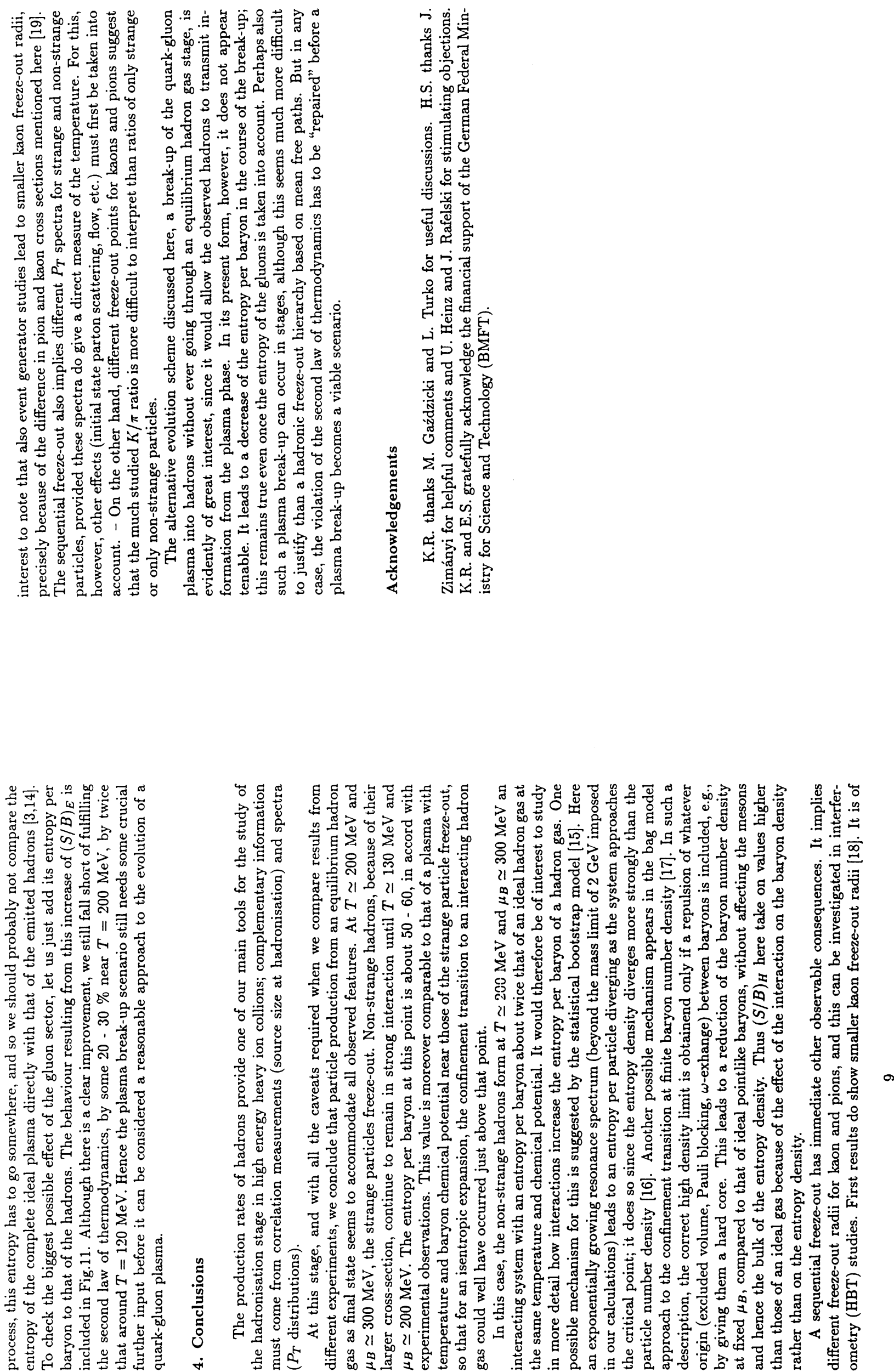

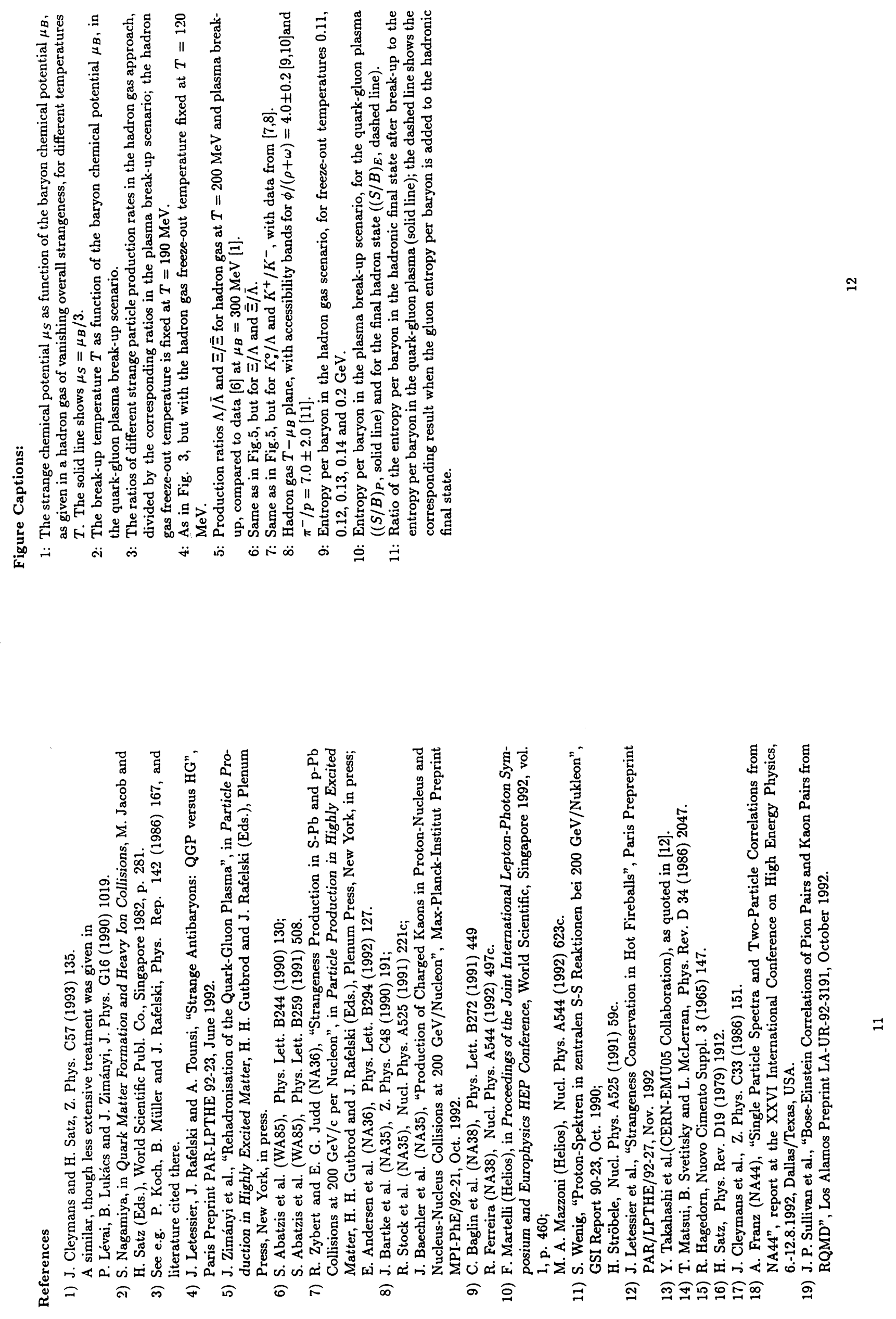

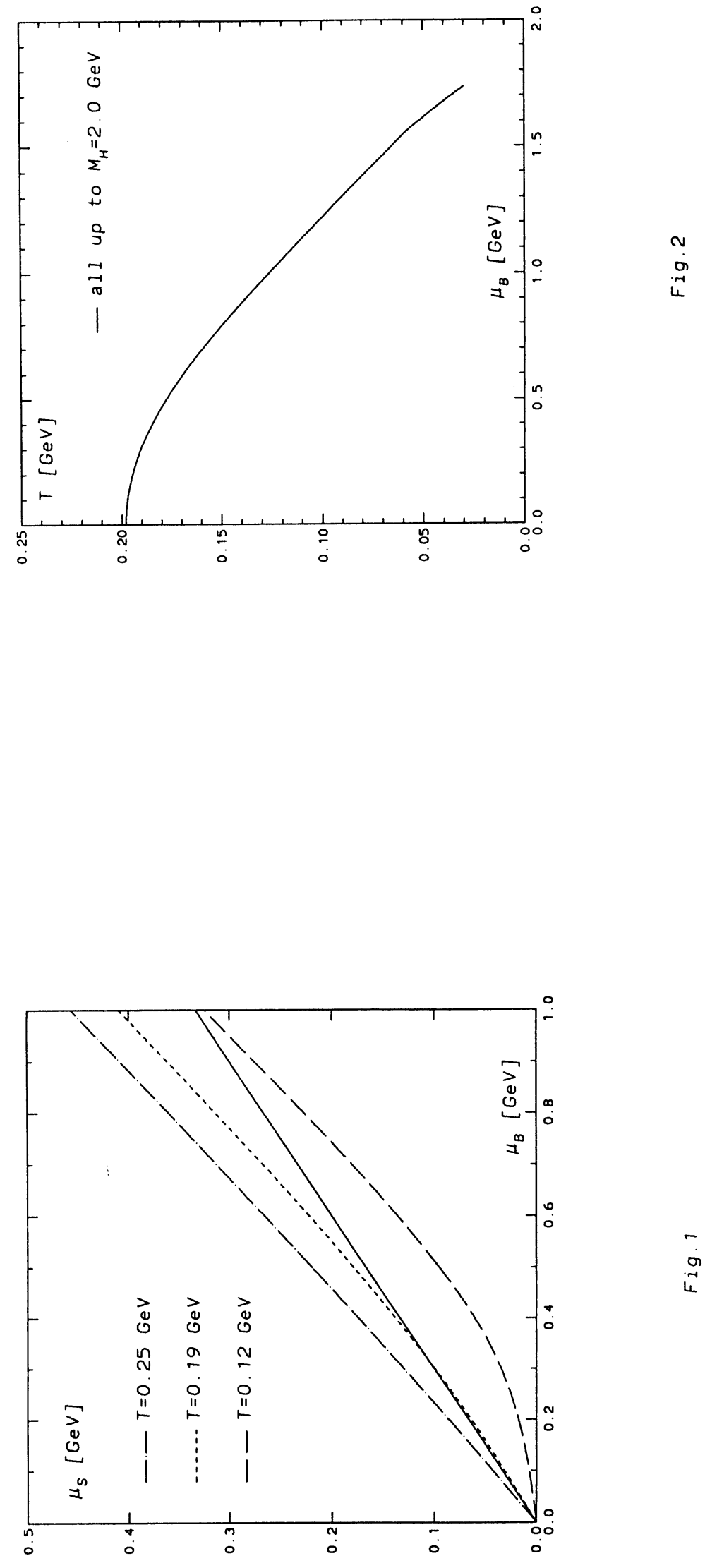


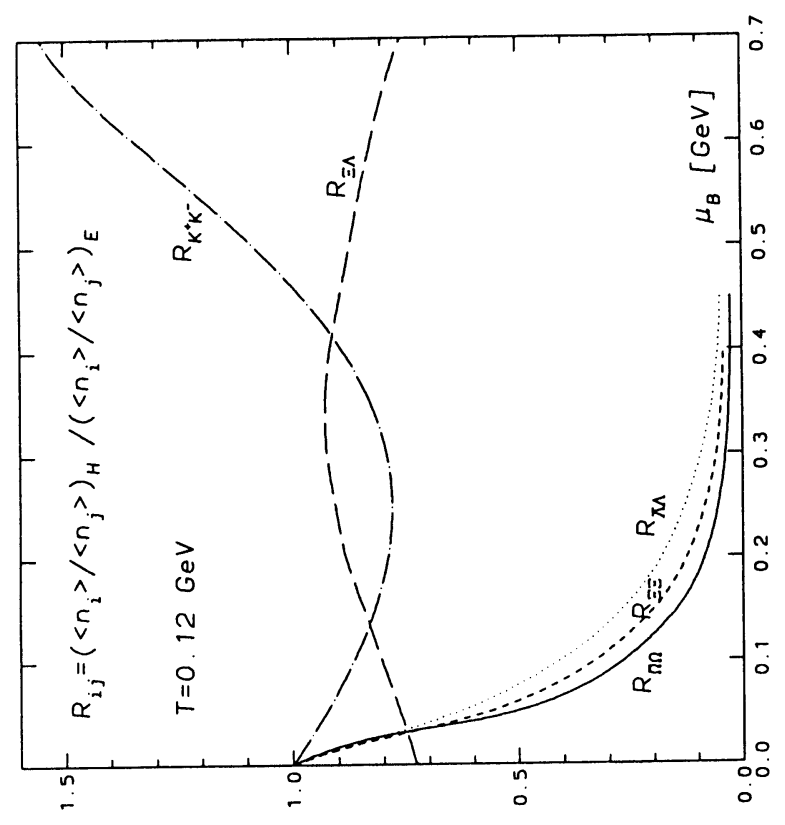

$\forall$
a
$\sim 1$

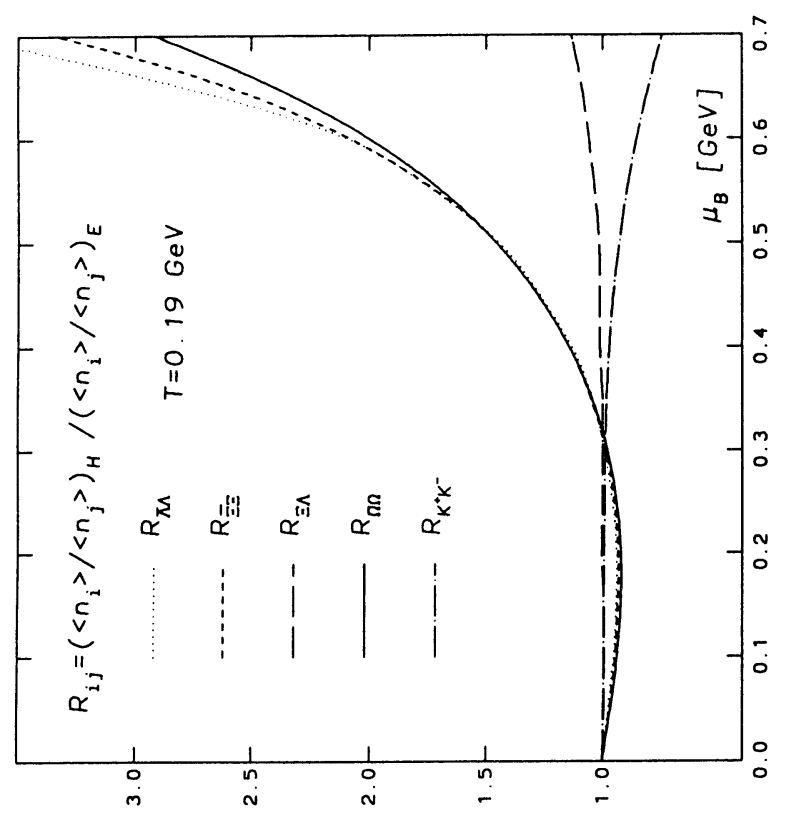

$m$
m.
Li 

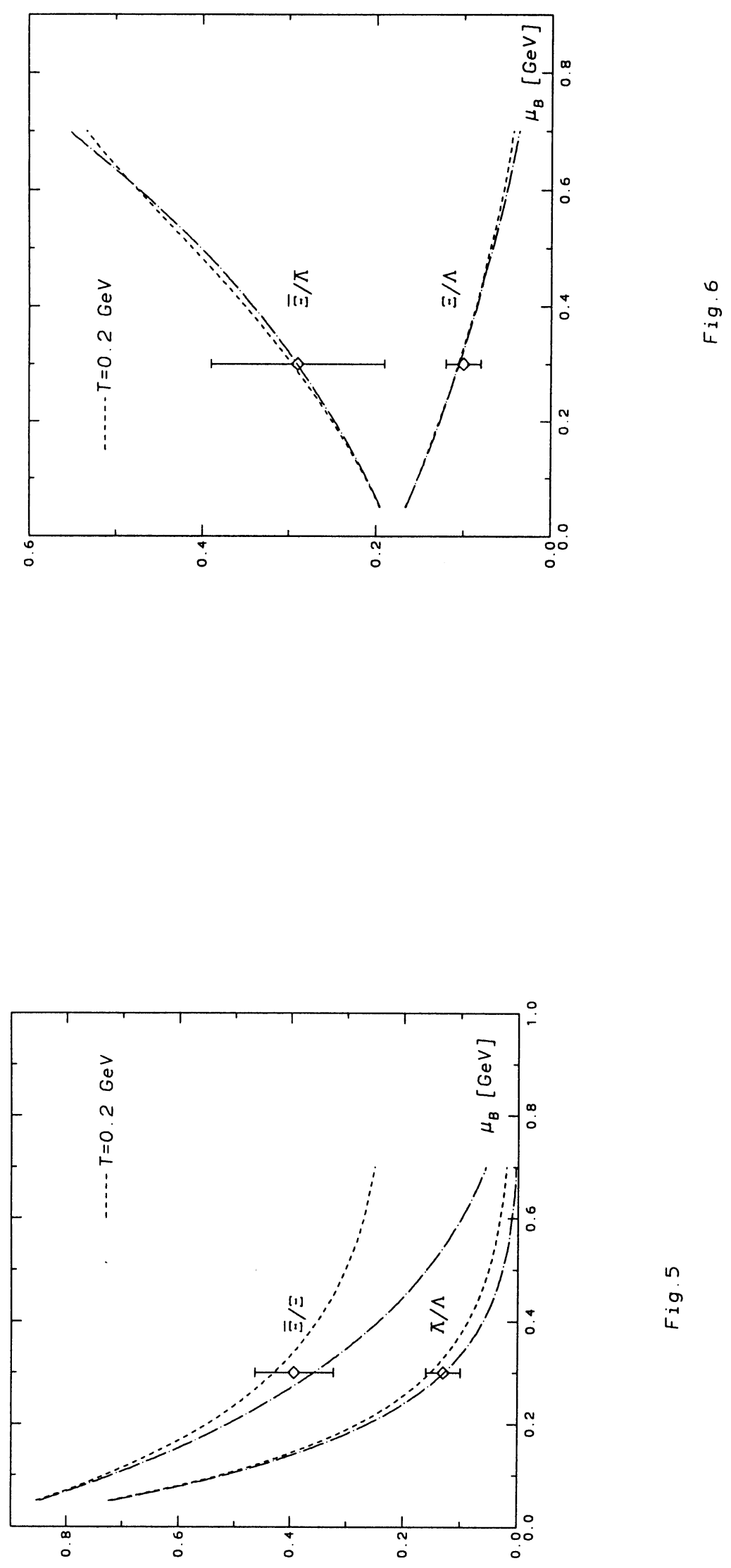

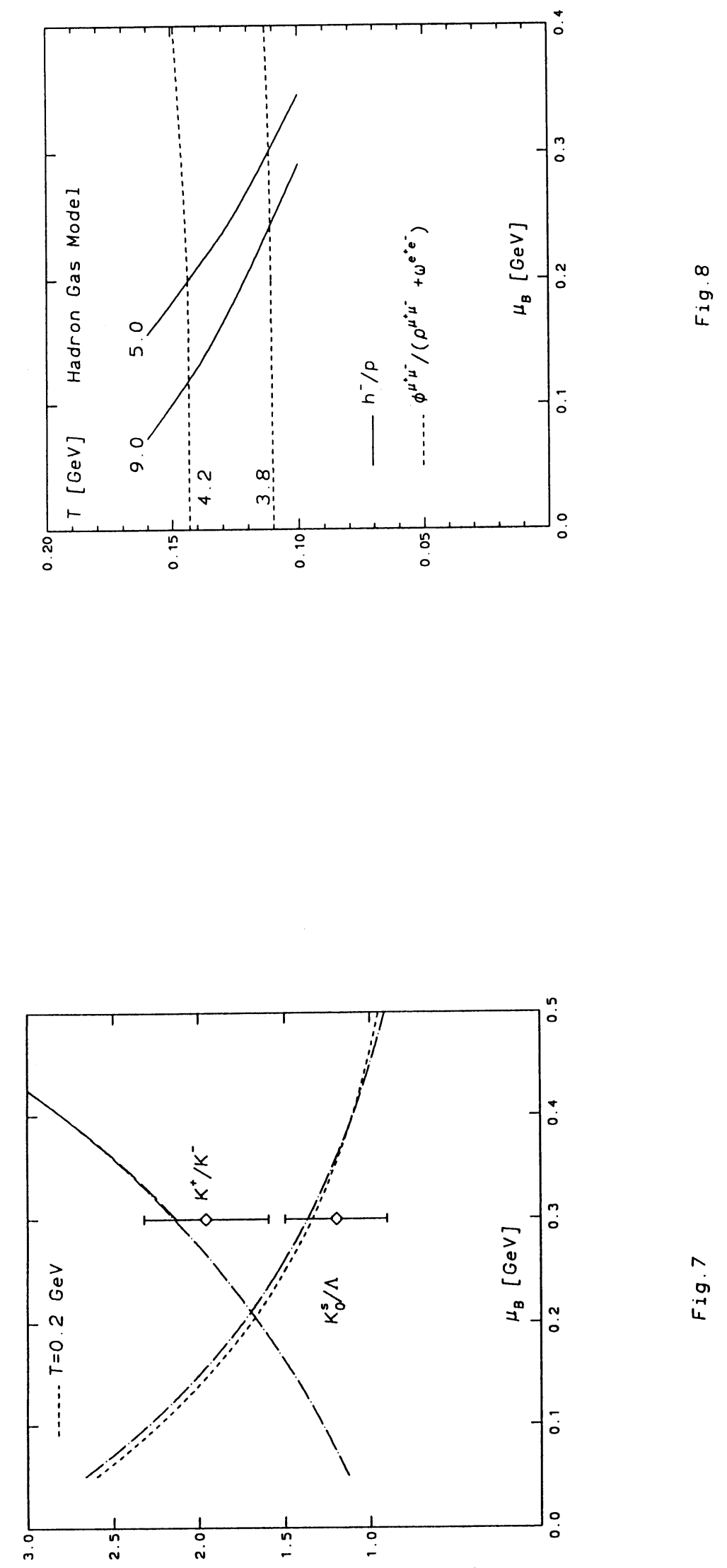

o
$i$
$i$ 

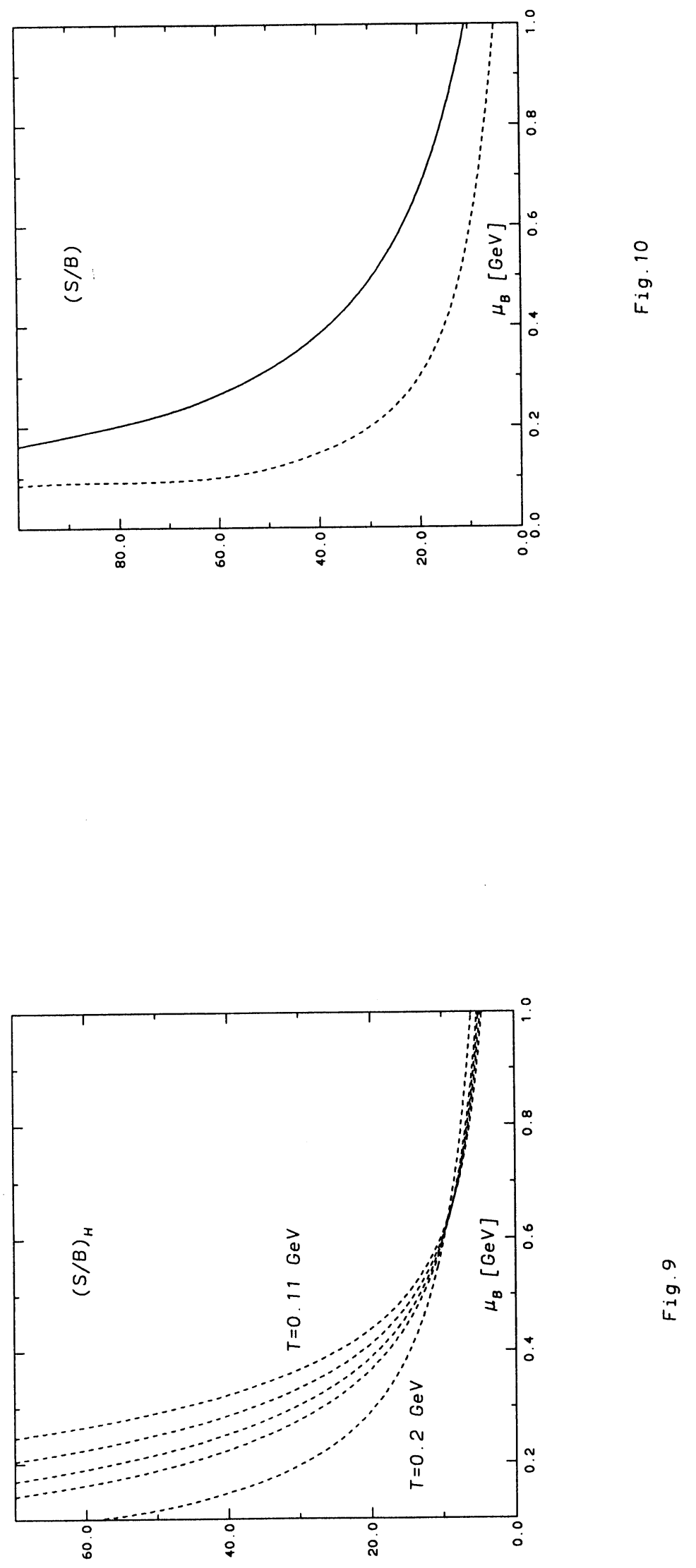


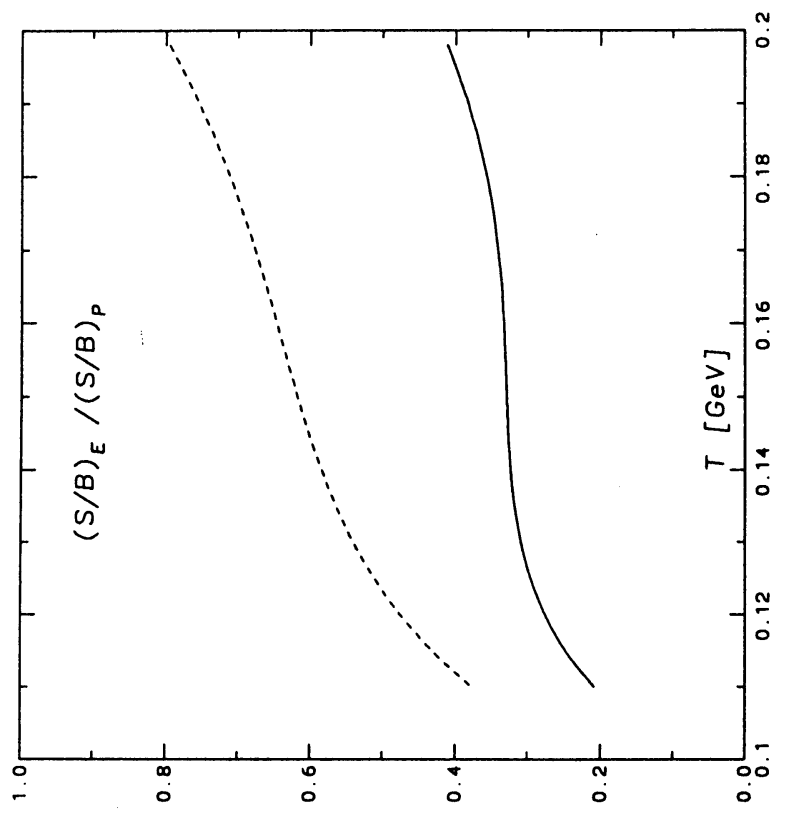

$=$ 